\title{
Avaliação de Desempenho Utilizando Diferentes Arquiteturas de Aprendizagem Profunda a Partir de Dados Relacionados a Precipitação Pluviométrica Coletados por Estação Meteorológica Automática
}

\author{
Roney Nogueira de Sousa ${ }^{1}$, Rhyan Ximenes de Brito ${ }^{1}$, \\ Janaide Nogueira de Sousa Ximenes ${ }^{2}$ \\ ${ }^{1}$ Instituto Federal de Educação, Ciência e Tecnologia do Ceará (IFCE) \\ Av. Tabelião Luiz Nogueira de Lima S/N - Tianguá - CE - Brasil \\ ${ }^{2}$ Faculdade IEducare (FIED) - Rua Conselheiro João Lourenço,
406 - CEP 62320-000 - Tianguá - CE - Brasil
\{nogueiraroney5 43, rxbrito, nogueirajanaide\} @gmail .com
}

\begin{abstract}
Rains play an important role in the social context as they bring the possibility of planning for the cultivation and harvesting of various food crops. Thus, the study aimed to predict rainfall through the application of Convolutional Neural Networks. The methodology used a database collected by an automatic meteorological station and the application of LeNet, VGGNet and ResNet architectures, as well as k-fold, SMOTE and z-score normalization techniques. The results showed that the VGGNet architecture had the best average hit rate with $91.53 \%$, while the specificity and false positive rate LeNet had the best results with $95.23 \%$ and $4.77 \%$ respectively.
\end{abstract}

Resumo. As chuvas exercem um importante papel no contexto social por trazerem a possibilidade de planejamento para o cultivo e colheita de várias culturas alimentares. Dessa forma o estudo teve como finalidade a predição de precipitações pluviométricas através da aplicação das Redes Neurais Convolucionais. Para a metodologia utilizou-se de uma base de dados colhida por uma estação meteorológica automática e da aplicação das arquiteturas LeNet, VGGNet e ResNet, assim como das técnicas k-fold, SMOTE e normalização z-score. Os resultados demonstraram que a arquitetura VGGNet obteve a melhor taxa média de acerto com 91,53\%, quanto a especificidade e taxa de falso positivo a LeNet obteve os melhores resultados com 95,23\% e 4,77\% respectivamente.

\section{Introdução}

As precipitações pluviométricas conhecidas como chuva são as formas mais baratas e ambientalmente adequadas de uso da água na agricultura, colaborando para a manutenção do equilíbrio dos recursos hídricos existentes em qualquer região [Dantas et al. 2016]. Assim observa-se que o desenvolvimento agrário de uma região está associado aos níveis de chuva, uma vez que a escassez é um fator desfavorável para o processo produtivo.

Por outro lado a utilização de algoritmos de aprendizagem profunda (AP) possuem como uma de suas vantagens a capacidade de aprendizagem em grandes quantidades de 
dados, o que os tornam uma importante ferramenta para a análise de grandes volumes de dados como os pluviométricos, principalmente partindo do conceito de que para as AP foram propostas novas ideias, originando assim as redes neurais profundas, como as Redes Neurais Convolucionais. O presente trabalho teve como objetivo principal realizar a predição de chuva com base em dados pluviométrica extraídos de uma estação meteorológica automática localizada em uma cidade do interior do estado do Ceará. Para o estudo foi utilizada uma base de dados, disponível em https://portal.inmet.gov.br/.

A metodologia utilizada baseou-se no estudo bibliográfico e na implementação das seguintes arquiteturas de redes neurais convolucionais: ResNet, LeNet e VGGNet, apoiadas em técnicas como validação cruzada $(k$-fold), balanceamento dos dados e na técnica SMOTE, em seguida realizou-se um comparativo entre os resultados baseados em procedimentos quantitativos realizados com base no treinamento e teste.

A motivação para este trabalho está relacionada a importância da aplicação de técnicas de Inteligência Artificial (IA) em situações que possam auxiliar profissionais da área da agricultura, oferecendo assim suporte de forma direta ou indireta na tomada de decisões no campo, pois tais informações afetam decisões relacionadas a irrigação, plantio, transporte, produção de proteína animal etc.

Este trabalho está dividido em cinco seções: a Seção 2 descreve alguns trabalhos relacionados, Seção 3 traz a fundamentação teórica sobre as arquiteturas escolhidas para serem trabalhadas, na Seção 4 é descrito os materiais e métodos utilizados. A Seção 5 apresenta os resultados e discussões, por fim a Seção 6 expoe as conclusões e trabalhos futuros.

\section{Trabalhos Relacionados}

Esta seção apresenta uma revisão bibliográfica de forma reflexiva sobre diferentes abordagens sobre o uso de técnicas de IA aplicada como ferramenta de auxílio na previsão climática.

[Kawase et al. 2015] utilizou a base de dados constituída da série histórica dos valores de precipitação total mensal nos meses do verão em Paty de Alferes obtida junto ao Instituto Nacional de Meteorologia, no período de 1993 a 2013. O modelo computacional utilizado para a predição de precipitação pluviométrica foi baseado em redes neurais de Perceptron de Múltiplas Camadas, com três camadas. O erro obtido pela rede MLP foi equivale a 3,2\% quando analisado o período de 2010 a 2013.

[Coutinho et al. 2016] apresenta uma abordagem computacional para a predição de um passo à frente em séries de dados meteorológicos pertencentes às regiões de Paty do Alferes e Paracambi. Para tanto, foram utilizados dois modelos de Redes Neurais Artificiais: Perceptrons de Múltiplas Camadas e Função de Base Radial. Apresentando resultados favoráveis entre $91 \%$ e $96 \%$ de acerto para todos os casos.

[da Costa Ferreira et al. 2019] buscou prever a precipitação de chuva do dia seguinte utilizando dados meteorológicos dos últimos 7 dias. Para cumprir tal objetivo foi avaliado diversos algoritmos de Redes Neurais Artificiais e Fuzzy para série temporal aplicando-os para predição de chuva. Dos vários algoritmos avaliados o LevengergMarguardt e Bayesian Regularization apresentaram menores desvios médios entre os valores observados e preditos. 
[dos Santos Sousa et al. 2017] realizou a utilização de redes neurais artificiais para previsão do volume de precipitações mensal na cidade de Manaus. Considerou dados de 1950 a 2015 e índices climáticos de temperatura da superfície do mar que influenciam a precipitação na região de interesse. Foram propostas, treinadas e testadas diferentes redes neurais para o problema em questão e, para cada mês do ano, foi selecionada a rede com melhor acurácia. Os resultados obtidos mostram a adequação dos modelos propostos, com taxa de acerto superior a $92 \%$.

Nesse contexto evidencia-se que independentemente da natureza das pesquisas a serem realizadas, os investigadores ao recorrerem às ferramentas digitais procuraram assegurar que os dados por si recolhidos tenham sido analisados de forma criteriosa e sistemática, gerindo mais eficazmente o tempo despendido e aumentando a confiabilidade dos resultados obtidos [Baugh et al. 2010].

\section{Fundamentação Teórica}

Esta seção apresenta uma revisão teórica sobre os assuntos abordados nesse trabalho com destaque para as redes neurais convolucionais ResNet, LeNet e VGGNet com suas particularidades.

\section{1. $\operatorname{ResNet}$}

A rede ResNet é uma estrutura robusta, que é capaz de alcançar um dos melhores resultados na área de redes convolucionais. Assim como dito por [Martins et al. 2019] a publicação dessa arquitetura de rede permitiu que fossem construídas e otimizadas várias redes muito mais profundas, com ela são alcançados desempenhos superiores as arquiteturas disponíveis até então. A Figura 1 mostra um exemplo da arquitetura de rede ResNet.

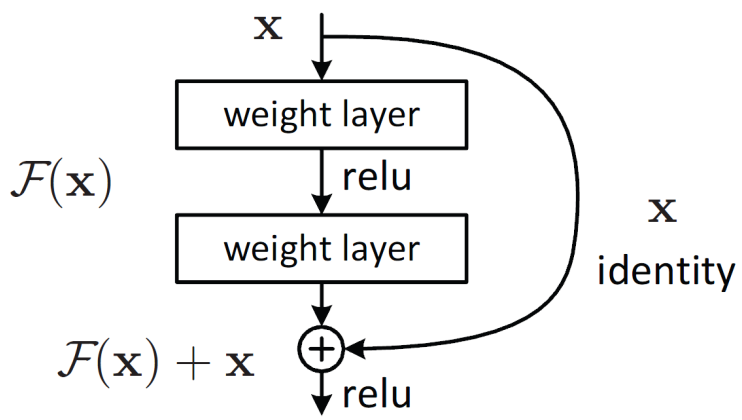

Figura 1. Arquitetura da Rede Neural Convolucional ResNet

Essa arquitetura trabalha na proposta em que as camadas continuem a receber os valores resultantes das funções de ativação Rectified Linear Unit, da camada anterior, mas também recebam os valores de entrada x dessas funções [Souza et al. 2020]. [Block et al. 2018] complementa ao afirmar que em algumas arquiteturas convencionas de redes neurais convolucionais o erro calculado é retro propagado e passado diretamente de uma camada para a próxima de forma linear, fazendo assim com que o gradiente seja recalculado em toda a rede. Porém em redes residuais como a ResNet são construídos certos tipos de atalhos que ignoram certas camadas da rede e passam o valor do gradiente mais para o início da rede sem que ele seja suavizado em inúmeras camadas. 


\subsection{LeNet}

A LeNet foi uma das primeiras redes neurais convolucionais que ajudaram a impulsionar o campo do Aprendizado Profundo. Este trabalho pioneiro de [LeCun et al. 1998] foi denominado LeNet5 após muitas iterações anteriores bem-sucedidas desde o ano de 1988 . Naquela época, a arquitetura LeNet era usada principalmente para tarefas de reconhecimento de caracteres, como leitura de Código de Endereçamento Postal (CEP), dígitos etc. [Bertoni and Feder 2018].

A arquitetura LeNet é uma rede neural convolucional composta por 7 camadas, a qual possui parâmetros ou pesos treináveis, proposta por [LeCun et al. 1998], inspirada no Neocognitron. Segundo [Bertoni and Feder 2018] a arquitetura LeNet possui uma estrutura simples e pequena, quando se trata de temos de memória ocupada, ela pode até mesmo rodar puramente em uma única CPU, tornando-a uma ótima arquitetura para aplicações envolvendo CNNs e sistemas embarcados. A Figura 2 exibe um exemplo da arquitetura LeNet utilizada no reconhecimento de imagens.

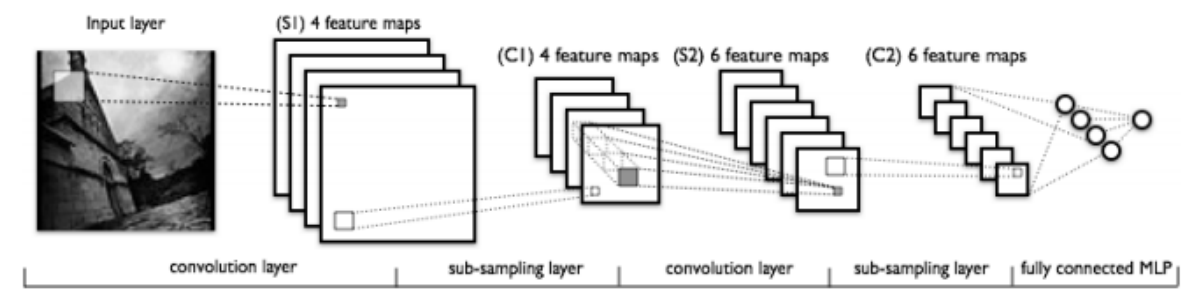

Figura 2. Arquitetura da Rede Neural Convolucional LeNet

\subsection{VGGNet}

A última das arquiteturas utilizadas na abordagem é a VGGNet, essa arquitetura foi proposta por [Chatfield et al. 2014], ela foi projetada para aumentar a profundidade das redes. A Figura 3 nos mostra um exemplo da arquitetura de rede ResNet.

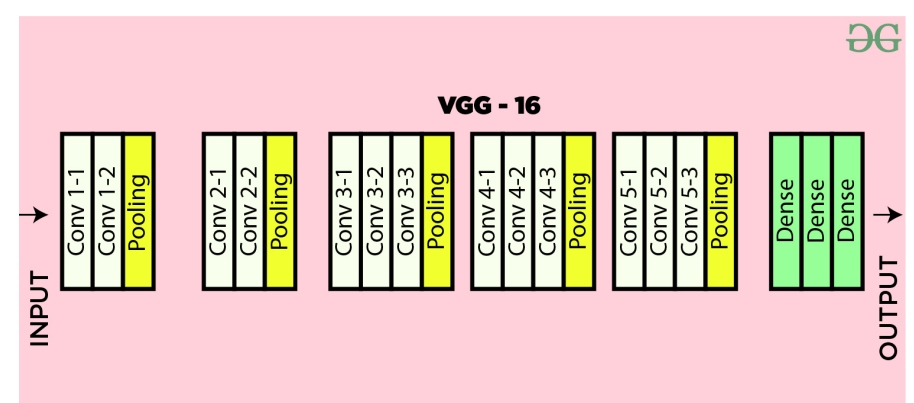

Figura 3. Arquitetura da Rede Neural Convolucional VGGNet

A VGGNet é composta principalmente por camadas de convolução, max pooling, completamente conectadas e Softmax. O fato de usar filtros de convolução com tamanho pequeno resultou em que não haja um aumento significativo de parâmetros a serem ajustados à medida que a rede cresce, desta forma, gerando eficiência [Cruz et al. 2019]. 


\section{Materiais e Métodos}

Para o emprego dos algoritmos utilizou-se da linguagem Python em sua versão 3.7 e uma base em dados pluviométrica extraída de uma estação meteorológica automática localizada em uma cidade do interior do estado do Ceará obtidos através do link: https://portal.inmet.gov.br/. Os dados utilizados foram coletados levando em consideração as informações obtidas durante o intervalo de 15/03/2018 a 11/05/2021. Salienta-se ainda que os mesmos passaram pelo processo de pré-processamento onde algumas amostras com atributos ausentes foram descartadas, assim como atributos que não estavam relacionados a classificação, além do descarte de informações redundantes.

A base de dados utilizada após o pré-precessamento, possui 23.879 amostras e 16 atributos como por exemplo, temperatura máxima, temperatura mínima, umidade máxima, umidade mínima pressão atmosférica, velocidade dos ventos, entre outros. Ressalta-se ainda que foram adicionadas a base de dados 22.436 amostras sintéticas através da técnica SMOTE. Quanto a variável desfecho, foi trabalhada com relação a ocorrência de chuvas na região. Assim as classes foram codificadas com "0"para ausência de chuva e "1"para presença de chuva. Para a classificado baseada nas arquiteturas utilizadas, salienta-se que foram testados vários hiperparâmetros de forma a ajustá-los através da estratégia Randomized Search à atingirem os melhores resultados.

A base de dados foi normalizada utilizando a técnica (z-score) e o balanceamento foi realizado através do método SMOTE garantindo assim a proporcionalidade entre as amostras utilizadas. Vale salientar que a geração dos dados sintéticos foi relacionada a classe minoritária a partir de vizinhos. Além disso foi utilizado o método de validação cruzada $k$-fold, com o $\mathrm{k}=10$ folds, o mesmo proporcionando os melhores resultados frente as demais configurações testadas para o mesmo.

\section{Resultados e Discussões}

Os resultados obtidos e analisados tiveram como base os percentuais da taxa média da acurácia adquiridos durante os treinamentos e testes realizados, foram também analisados os resultados das sensibilidades e especificidades.

Como observado na Tabela 1, o melhor caso da arquitetura LeNet atingiu uma acurácia de $92,49 \%$ de acertos e 7,51\% de erros e o pior caso $89,68 \%$ de acertos e 10,32\% de erros. A taxa média de acertos foi de $91,03 \%$ e $8,97 \%$ de erros. Para a especificidade a LeNet atingiu $95,23 \%$ e $4,77 \%$ de falso negativo.

Nota-se na Tabela 2 que o melhor caso da arquitetura ResNet atingiu uma acurácia de $90,34 \%$ de acertos e $9,66 \%$ de erros e o pior caso $87,56 \%$ de acertos e $12,44 \%$ de erros. A taxa média de acertos foi de $89,17 \%$ e $10,83 \%$ de erros. Para a especificidade a rede ResNet atingiu $90,25 \%$ e $9,75 \%$ de falso negativo.

Por fim na Tabela 3, observa-se que o melhor caso da arquitetura VGGNet atingiu uma acurácia de $92,75 \%$ de acertos e $7,25 \%$ de erros e o pior caso $90,36 \%$ de acertos e $9,64 \%$ de erros. A taxa média de acertos foi de $91,53 \%$ e $8,47 \%$ de erros. Para a especificidade a rede VGGNet atingiu $92,49 \%$ e $8,51 \%$ de falso negativo.

\section{Considerações Finais e Trabalhos Futuros}

$\mathrm{O}$ artigo relatou a utilização de redes neurais convolucionais para a previsão de precipitações pluviométricas em uma cidade do interior do Ceará com base em um banco 
Tabela 1. Resultados LeNet

\begin{tabular}{llcc}
\hline \hline Folds & Taxa de Acerto (\%) & Taxa de erro (\%) & Situação (Resultado) \\
\hline \hline 1 & 91,06 & 9,94 & \\
\hline 2 & 92,49 & 7,51 & Melhor Caso \\
\hline 3 & 90,55 & 9,45 & \\
\hline 4 & 91,60 & 8,40 & \\
\hline 5 & 90,45 & 9,55 & \\
\hline 6 & 89,68 & 10,32 & Pior Caso \\
\hline 7 & 90,06 & 9,94 & \\
\hline 8 & 92,26 & 7,74 & \\
\hline 9 & 91,70 & 8,30 & \\
\hline \hline-- & 90,53 & 9,47 & \\
\hline \hline
\end{tabular}

Tabela 2. Resultados ResNet

\begin{tabular}{llcc}
\hline \hline Folds & Taxa de Acerto (\%) & Taxa de erro (\%) & Situação (Resultado) \\
\hline 1 & 87,56 & 12,44 & Pior Caso \\
\hline 2 & 90,12 & 9,88 & \\
\hline 3 & 90,25 & 9,75 & \\
\hline 4 & 89,49 & 10,51 & \\
\hline 5 & 88,36 & 11,64 & \\
\hline 6 & 90,16 & 9,84 & \\
\hline 7 & 88,45 & 11,55 & \\
\hline 8 & 87,69 & 12,31 & \\
\hline 10 & 89,26 & 10,74 & \\
\hline \hline-- & 90,34 & 9,66 & Melhor Caso \\
\hline \hline
\end{tabular}

Tabela 3. Resultados VGGNet

\begin{tabular}{llcc}
\hline \hline Folds & Taxa de Acerto (\%) & Taxa de erro (\%) & Situação (Resultado) \\
\hline \hline 1 & 92,65 & 7,35 & \\
\hline 2 & 91,69 & 8,31 & \\
\hline 3 & 92,75 & 7,25 & Melhor Caso \\
\hline 4 & 90,56 & 9,44 & \\
\hline 5 & 91,83 & 8,17 & \\
\hline 6 & 90,36 & 9,64 & Pior Caso \\
\hline 7 & 92,16 & 7,84 & \\
\hline 8 & 91,31 & 8,69 & \\
\hline 9 & 91,62 & 8,38 & \\
\hline 10 & 90,36 & 9,64 & Pior Caso \\
\hline \hline-- & 91,53 & 8,47 & Caso Médio \\
\hline \hline
\end{tabular}


de dados extraídos de uma estação meteorológica automática levando em consideração as informações obtidas durante o intervalo de 15/03/2018 a 11/05/2021.

No estudo realizado percebe-se que a arquitetura LeNet e VGGNet obtiveram as melhores médias de acertos para as acurácias com 91,03\% e 91,53\% respectivamente, porém quando analisadas as taxas de especificidade e falso negativo pôde-se perceber que a arquitetura LeNet atingiu o melhor resultado com 95,23\% de especificidade e 4,77\% para o falso positivo enquanto a arquitetura $V G G N e t$ obteve $92,49 \%$ para a especificidade e 8,51\% para o falso positivo. Já a arquitetura ResNet obteve a pior média de acertos com $89,17 \%$, assim como os piores resultados para a especificidade e taxa de falso negativos.

Como trabalhos futuros sugere-se a utilização de outras arquiteturas de redes neurais convolucionais, com a finalidade de comparar os resultados de modo a descobrir qual arquitetura pode solucionar de forma mais eficaz o problema proposto.

\section{Referências}

Baugh, J. B., Hallcom, A. S., and Harris, M. E. (2010). Computer assisted qualitative data analysis software: a practical perspective for applied research. Revista Digital Del Instituto Internacional de Costos, (6):69-81.

Bertoni, A. L. and Feder, D. V. d. S. (2018). Rede neural convolucional aplicada à visão computacional para detecção de incêndio. B.S. thesis, Universidade Tecnológica Federal do Paraná.

Block, S. A. B. et al. (2018). Inspeção e classificação de picos em peças estampadas de metal utilizando rede neural convolucional. Master's thesis, Universidade Tecnológica Federal do Paraná.

Chatfield, K., Simonyan, K., Vedaldi, A., and Zisserman, A. (2014). Return of the devil in the details: Delving deep into convolutional nets. arXiv preprint arXiv:1405.3531.

Coutinho, E. R., Silva, R. M., and Delgado, A. R. S. (2016). Utilização de técnicas de inteligência computacional na predição de dados meteorológicos. Revista Brasileira de Meteorologia, 31:24-36.

Cruz, A. A. d. et al. (2019). Uma abordagem para reconhecimento de emoção por expressão facial baseada em redes neurais de convolução.

da Costa Ferreira, M., Rocha, J. V., Sannomia, D., and Ferreira, T. B. (2019). Método simplificado para previsão de chuva aplicado no agronegócio. In Congresso Transformação Digital 2019.

Dantas, D., Luz, T. M. O., de Souza, M. J. H., Barbosa, G. P., and Cunha, E. G. S. (2016). Uso de redes neurais artificiais na previsão da precipitação de períodos chuvosos. $R e$ vista Espinhaço-UFVJM, pages 11-18.

dos Santos Sousa, R., Silva, L., Guedes, E. B., and de Oliveira, M. B. L. (2017). Redes neurais artificiais aplicadas à previsão antecipada de precipitações na região central de manaus. IV Escola Regional de Informática Norte I, pages 1-9.

Kawase, K. H., Coutinho, E. R., and da Silva, R. M. (2015). Aplicação de modelo computacional de redes neurais na previsão de dados pluviométricos no município de paty alferes-rj. Proceeding Series of the Brazilian Society of Computational and Applied Mathematics, 3(1). 
LeCun, Y., Bottou, L., Bengio, Y., and Haffner, P. (1998). Gradient-based learning applied to document recognition. Proceedings of the IEEE, 86(11):2278-2324.

Martins, R. A. P. et al. (2019). Aplicação de redes convolucionais profundas para detecção de massas em mamografias.

Souza, V., Silva, L., Santos, A., and Araújo, L. (2020). Análise comparativa de redes neurais convolucionais no reconhecimento de cenas. Anais do Computer on the Beach, 11(1):419-426. 